Tecno Lógicas

ISSN 0123-7799

Vol. 18, No. 34, pp. 125-136

Enero-junio de 2015

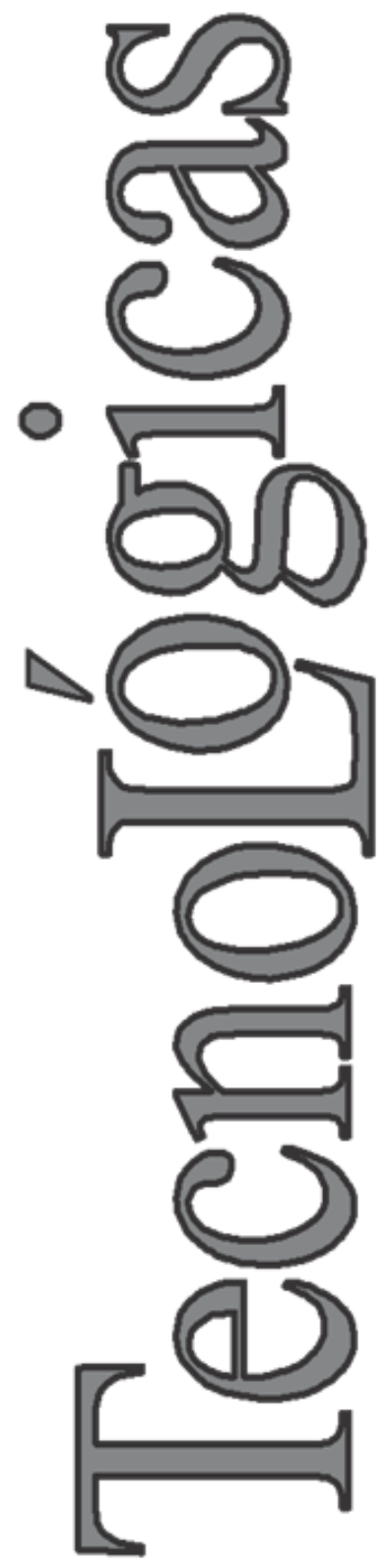

(C) Copyright 2015 por autores y Tecno Lógicas Este trabajo está licenciado bajo una Licencia Internacional Creative Commons Atribución (CC BY)

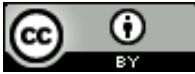

\section{Redes elásticas del futuro: beneficios para la red interna de telecomunicaciones de Colombia}

\section{Elastic networks of the future: benefits for the Colombian internal telecommunication network}

\author{
Andrés F. Betancur-Pérez ${ }^{1}$, Jhon J. Granada-Torres² \\ y Neil Guerrero-González ${ }^{3}$
}

Recibido: 30 de mayo de 2014, Aceptado: 15 de agosto de 2014

Cómo citar / How to cite

A. F. Betancur-Pérez, J. J. Granada-Torres y Neil Guerrero-González, "Redes elásticas del futuro: beneficios para la red interna de telecomunicaciones de Colombia”, Tecno Lógicas, vol. 18, no. 34, pp. 125-136, 2015.

1 M.Sc. en Telecomunicaciones, Departamento de Electrónica y Telecomunicaciones, Facultad de Ingeniería, Universidad de Antioquia, Medellín-Colombia, andresfbp@gmail.com

2 M.Sc. en Telecomunicaciones, Departamento de Electrónica y Telecomunicaciones, Facultad de Ingeniería, Universidad de Antioquia, Medellín-Colombia, jigranadat@gmail.com

3 Ph.D. en Fotónica, Departamento de Electrónica y Telecomunicaciones, Facultad de Ingeniería, Universidad de Antioquia, Medellín-Colombia, neguego@gmail.com 


\section{Resumen}

El continuo crecimiento de la demanda de datos está dirigiendo la investigación para dar luz a una red eficiente en el uso de la fibra óptica y el espectro radioeléctrico. La próxima generación de redes será el resultado de una convergencia entre el dominio óptico e inalámbrico para reunir cada una de sus virtudes en una vasta red de telecomunicaciones más resiliente y más simplificada. Colombia no estará marginada de estos avances ya que actualmente despliega fibra óptica por gran parte del territorio nacional y por tal motivo debe estar informada de los progresos actuales en lo referente a redes de telecomunicaciones de nueva generación y orientar de mejor forma los lineamientos legales del ministerio de TIC (Tecnologías de la Información y las Comunicaciones) para traer en consecuencia grandes beneficios a la regulación de la red interna de telecomunicaciones en cuanto a lo decretado acerca de los inmuebles de propiedad horizontal. En este trabajo se plasman los avances en investigación concernientes a transmisiones por fibra óptica, incluyendo el nuevo paradigma de redes híbridas de fibra óptica con redes inalámbricas que permitirán simplificar la red global de telecomunicaciones. Las redes elásticas del futuro sugieren que las redes internas de telecomunicaciones serán más reducidas, simples y versátiles, lo cual facilitará la libre competencia entre los proveedores de servicios de comunicaciones, evitando el monopolio de espacios dentro de edificios de propiedad horizontal.

\section{Palabras clave}

FI-WI, PON, redes elásticas, redes malla, supercanales, WDM.

\section{Abstract}

The continuous growth in data demand is leading research to bring to light an efficient network using fiber optics and radioelectric spectrum. The next generation of networks will be the result of a convergence between the optical and wireless domain with the purpose to gather all of their virtues in a vast telecommunications network more resilient and more simplified. Colombia will not be marginalized of these advances because actually has deployed optical fiber in most of the country and therefore they must be informed of current progress in terms of new generation of telecommunications networks and guide in a better way the legal guidelines of the Ministry of ICT (Information and Communications Technology) to bring great benefits to the regulation of internal telecommunications network, concerning what was decreed about the immovable of horizontal property. In this paper, research advances are reflected concerning optical fiber transmission, including the new paradigm of hybrid fiber optic networks with wireless networks that will simplify the global telecommunications network. The elastic networks of the future suggest that internal telecommunications networks will be smaller, simple, and versatile, which will facilitate competition between communication service providers, avoiding the monopoly of spaces within horizontal property buildings.

\section{Keywords}

Elastic networks, FI-WI, mesh networks, PON, superchannels, WDM. 


\section{INTRODUCCIÓN}

El estado colombiano en el marco de la ley 1341 del 2009, en su actividad de motivar la inversión e innovación de nueva infraestructura de telecomunicaciones tiene presente que gran parte de la nueva infraestructura que debe ser desplegada para contar con una red nacional de datos de banda ancha, se debe lograr con el despliegue de nuevas redes o mediante actualizaciones de las existentes para garantizar que la utilización y la remuneración de esta acelere la inversión y la innovación [1]. Por lo anterior, los agentes reguladores deben propiciar el entorno adecuado para la implementación de redes que permitan actualizaciones y mejoras de capacidad de transmisión de datos para ajustarse al perfil dinámico de la demanda de información. Por lo tanto, para Colombia es de vital importancia tener presente los desarrollos tecnológicos llevados a cabo por la comunidad científica para facilitar la evolución de las redes de telecomunicaciones en el país [2] hacia un concepto de red elástica para utilizar eficientemente el espectro limitado de los canales de transmisión, reutilizar la infraestructura existente y propiciar un entorno simplificado que permita la fácil participación de los proveedores de servicio de telecomunicaciones.

La investigación en temas de telecomunicaciones ha dado pasos rápidos en la solución del problema de reutilización del espectro de los canales de telecomunicaciones [3], [4] y entre estos están los Supercanales, que gracias a los avances en DSPs (Digital Signal Processor) puede modificar su capacidad y recursos de acuerdo con las demandas latentes de información [5]-[10]; las redes WDM/PON que permitirán no solo compartir el ancho de banda entre los usuarios sino también las longitudes de onda bajo demanda o criterios de calidad de servicio [11]-[15]; el paradigma Fi-Wi que gracias a los adelantos en la tecnología radio sobre fibra (RoF) y redes LR-PON, que permitirán la consolidación de la red metropolitana y la red de acceso, además de una mayor capacidad, ubicuidad, resiliencia y un mejor uso del espectro radioeléctrico y el ancho de banda de la fibra óptica [16]-[20].

El artículo se estructura de la siguiente manera: primero se aborda el tema de las redes de acceso ópticas de próxima generación. Luego se tratará la convergencia FIWI. A continuación se trata el tema de la elasticidad en las redes de transporte desde el punto de vista de la tecnología de los Supercanales. Por último se analizan los beneficios que tendría Colombia con la implementación de las redes elásticas y se concluye.

\section{REDES PON DE PRÓXIMA GENERACIÓN}

Las redes PON son redes de arquitectura punto-multipunto que surgen como una respuesta tecnológica a la necesidad de disponer redes de acceso de banda ancha a un costo reducido para satisfacer la creciente demanda de información de los usuarios de Internet. Su reciente aparición se debe a la necesidad de aumentar el ancho de banda para el soporte de servicios video-céntricos a los usuarios finales. Investigaciones recientes, muestran que las redes PON están penetrando rápidamente en la vida de los usuarios residenciales y empresariales [21], [22] ya que reducen gratamente el costo de operación de la red y el costo de capital. Sin embargo, en los próximos años, la reducción de los costos de las redes PON no serán suficientes, e.g., al Reino Unido le costaría 15 billones de libras esterlinas desplegar redes de acceso basadas en fibra [22]. Esto lleva a replantear para el futuro las redes de acceso ópticas de banda ancha.

\subsection{Redes WDM/PON}

En los próximos años se pronostica un incremento del tráfico y es por tal motivo 
que WDM/PON resulta atractivo como una tecnología a prueba de futuro [23], [24]. La arquitectura de una red WDM/PON se ilustra en Fig. 1.

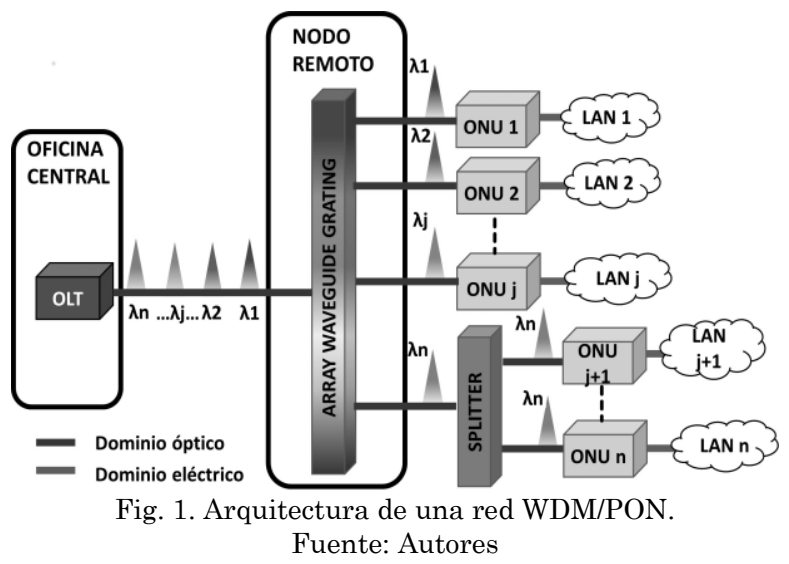

WDM/PON es una red que provee un acceso al medio a través de la multiplexación por división de longitud de onda. Como se puede apreciar en la Fig. 1, la OLT (Optical Line Terminal) provee un conjunto de longitudes de onda que viajan sobre la misma fibra hasta el multiplexor denominado AWG (Array Waveguide Grating) que se encarga de dividir la señal y enviar cada longitud de onda a su respectiva ONU (Optical Network Unit) destino. Una característica a tener en cuenta de WDM/PON es que se comporta como un conjunto de redes PON basado en TDM (Multiplexación por División de Tiempo: Time Division Multiplexing), ya que se puede conectar después del AWG un Splitter (o Divisor de potencia) para subdividir la longitud de onda entre otro subgrupo de usuarios mediante TDM. Este tipo de redes posee dos enfoques como lo ilustra la Fig. 2 [25].

En el enfoque descrito en la Fig. 2a se emplea un AWG que distribuye a las ONUs respectivas las longitudes de onda. En el enfoque descrito en la Fig. 2b se da uso del Splitter de potencia de las redes PON actuales ya que estos no dependen de la longitud de onda y es un enfoque atractivo porque se puede adaptar fácilmente a la infraestructura actualmente desplegada [25].

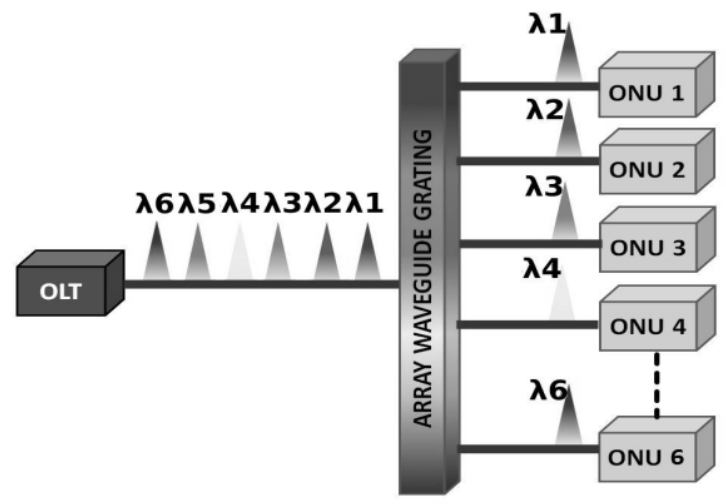

WDM/PON ESTÁTICO
- Es una buena opción para aplicaciones
de largo alcance
- Asignación estática de longitudes de onda

a)

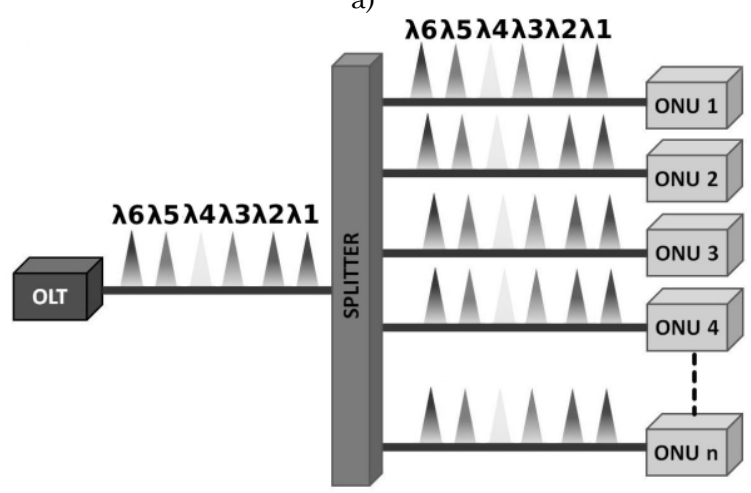

WDM/PON DINÁMICO

- Es una buena opción para aplicaciones de corto alcance

- Asignación dinámica de longitudes de onda

b)

Fig. 2. Enfoques de WDM/PON. Fuente: Autores

Para habilitar la asignación dinámica de longitudes de onda es necesario tener presente dos elementos claves: un enrutador de longitudes de onda reconfigurable y un protocolo en la capa de enlace que lo controle. En la literatura se han trabajado en varios protocolos para la asignación dinámica de longitudes de onda y ancho de banda en redes WDM/PON. Protocolos como el SIPACT (Simultaneous Interleaved Polling with Adaptive Cycle Time) y WDMIPACT (WDM Interleaved Polling with Adaptive Cycle Time) han sido demostrados teóricamente y presentan un gran desempeño en cuanto a la latencia de la red [26]-[28]. El esquema de WDM/PON planteado en Fig. $2 \mathrm{~b}$ en el futuro cercano 
será el más apto candidato para suministrar conectividad eficiente en el acceso para dar un uso óptimo del ancho de banda disponible de la fibra óptica al asignar dinámicamente las longitudes de onda según la demanda de cada usuario, lo cual permitirá una mayor consolidación de la infraestructura de las redes en Colombia y permitirá que estas perduren durante un horizonte de tiempos más prolongado para soportar la creciente demanda de información.

\subsection{Redes LR-PON}

Existe una alternativa a las redes PON convencionales y es una nueva tecnología denominada Long Reach Passive Optical Network (LR-PON) o red PON de largo alcance, que aprovecha las virtudes de los amplificadores ópticos y la tecnología WDM para lograr distancias de $20 \mathrm{~km}$ $100 \mathrm{~km}$ y la consolidación de las OLT y varias oficinas centrales de un proveedor de servicio lo cual reduce los costos de operación OpEx. LR-PON al brindar mayor cobertura geográfica, posibilita combinar las redes de acceso y metropolitanas en una sola red integrada, lo cual además brinda una reducción en el costo de propiedad CapEx y simplifica la red de telecomunicaciones [29].

En la Fig. 3 se expone la arquitectura LR-PON. En [29], se describen fenómenos que deben investigarse para tener acceso a las virtudes de LR-PON relacionados al uso de amplificadores, a la topología y a las largas distancias.

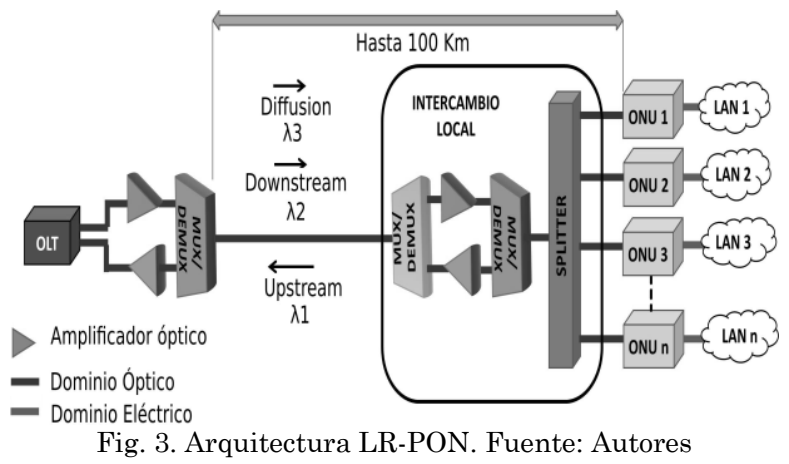

Aun así se ha demostrado su potencial en varios países y entre los más destacados se encuentran las realizadas por ACTSPLANET (Advanced Communication Technologies and Services - Photonic Local Access NETwork) con tamaños de división de 2048 ONUs, enlaces de $100 \mathrm{~km}, 2,5$ Gbps en downstream (Flujo de bajada) y 311 Mbps en upstream (Flujo de Subida) usando como medio de amplificación en upstream los SOAs (Amplificadores Ópticos de Semiconductor: Semiconductor Optical Amplifier) y EDFAs (Amplificadores de Fibra Dopada con Erbio: Erbium Doped Fiber Amplifiers) en downstream, para una relación señal a ruido (SNR: Signal to Noise Ratio) requerido de $18,6 \mathrm{~dB}$ y un BER (Tasa de Error de Bits: Bit Error Rate) de $10^{-9}$ con modulación OOK y $3 \mathrm{~dB}$ de margen de potencia óptica [30]-[32]. Las redes LR-PON tendrán la capacidad de reconfigurar la ganancia del amplificador según lo requiera una OLT ya que cada ONU estará a una distancia diferente con respecto a la OLT. Por otro lado adoptará el comportamiento dinámico de las redes $\mathrm{WDM} / \mathrm{PON}$.

\section{CONVERGENCIA FI-WI}

La necesidad de tener una plataforma única de comunicaciones cableada e inalámbrica que permita transmitir diferentes servicios ha motivado a los operadores a emplear tecnologías que soporten la convergencia de los servicios. Adicional a esto, se espera, que dicha plataforma proporcione gran capacidad y movilidad a los usuarios finales mediante el uso de la fibra óptica para cubrir extensas áreas geográficas y brindando acceso a la red con tecnologías inalámbricas como WiFi (wireless fidelity) o LTE (Long Term Evolution), dando lugar a un nuevo paradigma denominado Fi-Wi (Fiber-Wireless). Estas redes brindan acceso a la red usando tecnologías inalámbricas hacia puntos fijos que se enlazan a la oficina central mediante una 
red LR-PON. En la Fig. 4 se puede ilustrar que en la plataforma Fi-Wi la fibra óptica une la OLT con la ONU de igual forma que en PON, y las ONU están conectadas con dispositivos denominados gateways que se encargan de enviar y recibir señales de un conjunto de enrutadores inalámbricos configurados en una topología de malla [33].

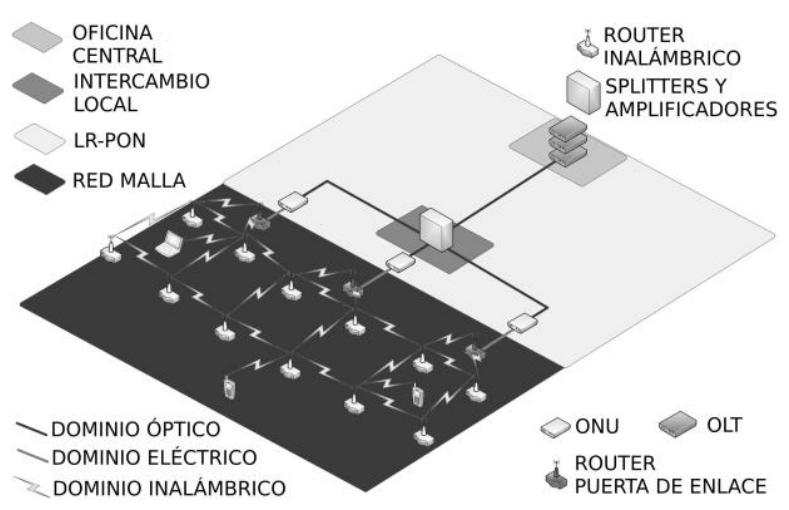

Fig. 4. Arquitectura de una red Fi-Wi. Fuente: Autores

Entre las ventajas que brindan las redes Fi-Wi se encuentran: la consolidación de la infraestructura lo cual implica una reducción en el costo de propiedad y operación; simplicidad que como consecuencia facilita la administración de la red; ahorro de instalación de cables hasta el usuario final que implica una reducción de la contaminación visual y costos; la ubicuidad que brinda la flexibilidad al usuario de la red de utilizarla donde y cuando lo necesite; robustez en la protección de la red en caso de fallas ya que la topología de malla brinda redundancia al disponer de los dispositivos de la malla para encontrar nuevos caminos hacia el destino; el ahorro de energía y esto es debido a que se podrían deshabilitar aquellas interfaces inalámbricas que tengan poca carga de trabajo o no estén realizando tarea alguna y en caso contrario habilitarlas en horas de mayor tráfico [33]-[35].

Las características previas brindarán a la red de acceso FI-WI una mayor reconfigurabilidad y esto es debido a que los dispositivos de radiofrecuencia en la actuali- dad poseen algo de comportamiento elástico; la asignación de portadoras de RF, deshabilitar transmisores, asignar capacidad y asignar rutas alternativas son ahora características comunes en dispositivos de comunicación inalámbrica eléctrica. El mayor reto en cuanto a redes FI-WI es habilitar el enrutamiento de redes malla y la tecnología en interfaces de Radio sobre Fibra.

Actualmente, implementar Fi-Wi es posible gracias a la tecnología en desarrollo de Radio sobre Fibra (RoF: Radio over Fiber) [36]. Un sistema RoF consiste en transmitir señales de radiofrecuencia directamente por la fibra óptica hacía las estaciones base (BS: Base Station), en las cuales se efectúa una conversión ópticaeléctrica para enviar la señal al medio, sin necesidad de generar portadoras de frecuencia, independiente del protocolo de la red, lo que reduce costos, teniendo BS con bajo consumo de potencia, de esta manera, RoF centraliza los equipos más complejos en la CO (Oficina Central: Central Office), permitiendo un fácil mantenimiento de la red [37].

Actualmente se espera utilizar el espectro de la banda de las ondas milimétricas $(30$ - $300 \mathrm{GHz})$ donde se dispone de un mayor ancho de banda. El estándar Wireless $\mathrm{HD}$ opera a $60 \mathrm{GHz}$ y logra transmisiones alrededor de 5 Gbps y se espera que logre hasta los $20 \mathrm{Gbps}$, de igual manera, es de interés para los sistemas RoF, la banda ubicada en el rango de los 75 y 110 $\mathrm{GHz}$, denominada banda $\mathrm{W}$, debido a que presenta una ventana de transmisión más amplia, con pérdidas mínimas de propagación, y sería más adecuada para aplicaciones multiGbps en exteriores en un futuro cercano [38]. En [39] se propone una red que opera en la banda $\mathrm{W}$ para velocidades de hasta 40 Gbps. Recientemente, se han logrado transmisiones de 2,5 Gbps sobre 40 $\mathrm{km}$ para un sistema de RoF full-duplex con menos de $2 \mathrm{~dB}$ de atenuación de potencia, en la banda de ondas milimétricas [36]. 


\section{ELASTICIDAD EN REDES DE TRANSPORTE}

Las redes de transporte se transformarán de un concepto rígido a uno elástico como los denominados Supercanales. Los sistemas WDM operan en rejillas fijas con separaciones de $12,5,25,50$ y $100 \mathrm{GHz}$ según las recomendaciones de la ITU-T. Esto restringe el ancho espectral de cada canal, el cual además debe tener una banda de guarda para evitar interferencia con canales adyacentes [40]. Para aumentar la capacidad de transmisión, con tasas por canal superiores a los 100 Gbps, los Supercanales proponen aumentar la eficiencia espectral, primero, por medio de los formatos de modulación, aumentando la cantidad de símbolos por unidad de tiempo, para lograr interfaces de 1 Tbps.

Investigaciones recientes presentan formatos de modulación de grandes constelaciones como PM-1024QAM [3]. No obstante, el aumento de formatos de modulación en el dominio óptico está restringido por el rápido incremento del valor de penalidad de la relación señal a ruido, lo que a su vez restringe al sistema a tener transmisiones de cortas distancias ya que aumentar la potencia está limitado por los efectos no lineales propios de la fibra óptica que afectan la transmisión, y además en el futuro los sistemas tendrán como condición ser eficientes en el consumo de potencia siguiendo la línea de las tecnologías "Green" [40]-[45].

Otra forma de incrementar la capacidad es utilizar sistemas multiportadora en donde cada subportadora será modulada por formatos de modulación avanzado como BPSK (Binary Phase Shift Keying), QPSK (Quadrature PSK) y mQAM (m Quadrature Amplitude Modulation) y mediante pulsos de Nyquist, logrando una alta eficiencia y densidad en el espectro lo cual permitirá escalar la tasa neta de transmisión, según lo imponga la demanda de información [46]-[48]. Lo anterior sugiere el empleo de dispositivos ópticos con anchos de banda y frecuencia central sintonizable para habilitar los Supercanales ya que las bandas de guarda en cada reconfiguración se desplazarán en el dominio de la frecuencia tal y como se observa en la Fig. 5 [5], [49].

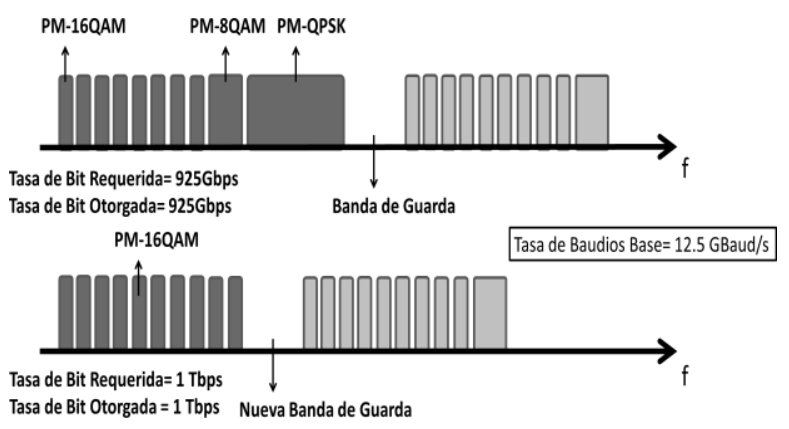

Fig. 5. Capacidad y espaciamiento entre canales reconfigurable. Fuente: Autores

La reconfiguración de los formatos de modulación no solo permitirá el concepto de consumo de capacidad bajo demanda, sino también adecuar la señal a las condiciones de la fibra óptica como la longitud, dispersión cromática y dispersión por modo de polarización. En esencia, una señal desde su nodo de origen hasta su nodo destino, puede adquirir entre un par de nodos varios formatos de modulación basándose en la distancia de los enlaces para mejorar las características de propagación como el OSNR (Relación Señal a Ruido Óptico) a través de la fibra óptica (ver Fig. 6) [5]. Incluso de acuerdo al estado del tráfico en la red se puede modificar el formato de modulación a uno que mejore la sensibilidad del receptor con menos potencia de lanzamiento para disminuir la energía utilizada para la transmisión [50].

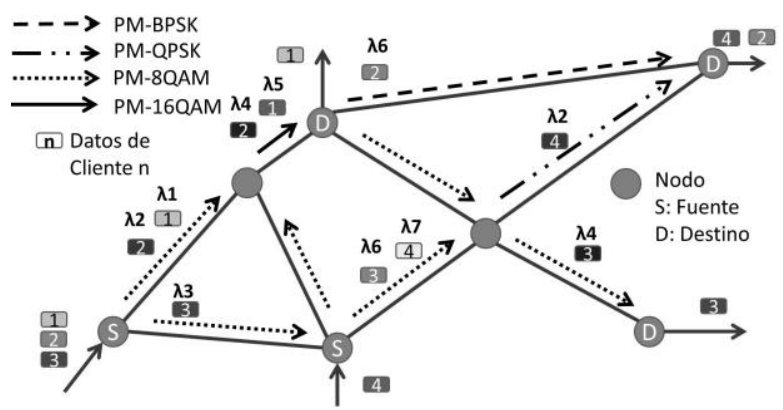

Fig. 6. Red óptica elástica. Fuente: Autores 
En la Fig. 6 se puede observar cómo cambian de canal (distinguidos como $\lambda \mathrm{n}$ ) los conjuntos de longitudes de onda (distinguidas por números) entre los nodos origen y destino. Esto posibilita a la red entera reutilizar longitudes de onda en algunas trayectorias donde no se utilizan y asignarlas de manera eficiente en la etapa de diseño de la red o por alguna falla presentada como cortes de fibra óptica o fallas en los dispositivos de toda la trayectoria implicada. En el contexto de los Supercanales, esta función debe habilitarse para optimizar el uso de los recursos de la red óptica como el ancho de banda y las longitudes de onda, y un dispositivo que puede cubrir tales requerimientos son los conversores de longitud de onda netamente ópti$\cos$ [51]-[54].

\section{BENEFICIOS DE LAS REDES ELÁSTICAS EN RITEL DE COLOMBIA}

En Colombia, el ministerio de TIC ha evidenciado un gran incremento en la solicitud de servicios de comunicaciones móviles o cableadas. Este incremento hace necesario un reglamento para la red que va entre el inmueble que va desde el edificio con régimen de propiedad horizontal donde yace el prestador de servicios hasta el inmueble del usuario final. Dicho reglamento está orientado a facilitar el ingreso de varios proveedores de servicios al interior de los inmuebles de propiedad horizontal para que exista una libre competencia y así promover una mejora en los servicios para el usuario final. La reglamentación entonces mitiga los cuellos de botella que se presentan cuando un proveedor llega primero al interior de una propiedad horizontal y dificulta el ingreso de otros prestadores de servicios. Por otro lado, se reglamenta la construcción de inmuebles ya que en Colombia se diseñan en su mayoría para el paso de una sola red interna de telecomunicaciones.

La red interna de telecomunicaciones comprende todos los equipos activos y pasivos de telecomunicaciones que yacen dentro de las instalaciones de inmuebles sometidos al régimen de propiedad horizontal. En la Fig. 7 se puede observar el esquema general de una red interna de telecomunicaciones.

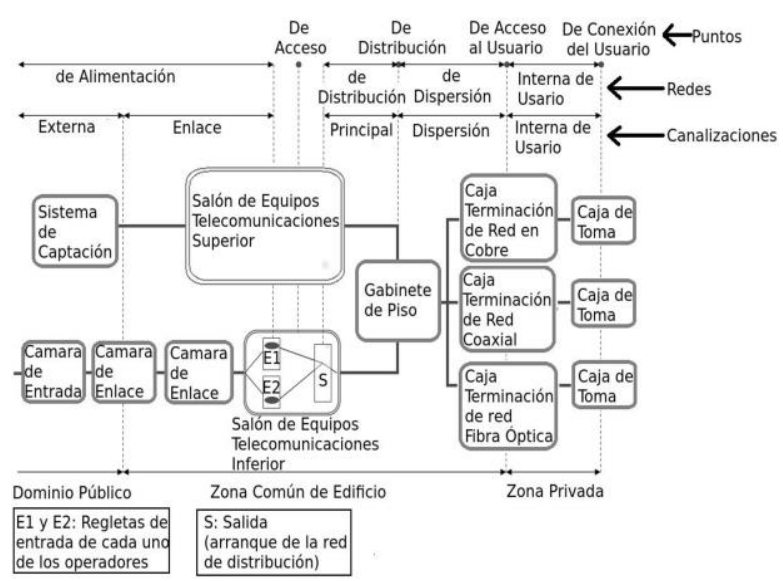

Fig. 7. Esquema general de una red interna de telecomunicaciones. Fuente: [55]

La red interna la comprenden 3 niveles que son: el dominio público, zona común del edificio y zona privada. El dominio público consta de la red pública de telecomunicaciones que llegan hasta las edificaciones en régimen de copropiedad. La zona privada consta del frente de equipos que se conectan a los terminales de los usuarios finales. En la zona común del edificio yacen dos cuartos principales conocidos como RITS (Recinto de Instalaciones de Telecomunicaciones Superior) y RITI (Recinto de Instalaciones de Telecomunicaciones Inferior). En RITS se alojan todos los elementos activos y pasivos que se encargan de procesar las señales del sistema de captación de transmisiones radiodifundidas y distribuirlas a las redes privadas. En RITI se alojan los elementos de terminación de cables como los distribuidores eléctricos, Strips telefónicos y distribuidores ópticos, que conectan la red pública aérea o subterránea con los dispositivos de la red privada de cada proveedor. Por otro lado se alojan algunos elementos activos como multiplexores, concentradores, dispositivos de 
red $y$ equipos de conversión optoelectrónica. Al dimensionar la cantidad de usuarios a los que un proveedor sirve, es de esperarse que la red interna de telecomunicaciones en la zona común del edificio sea considerablemente compleja ya que en los edificios de régimen de propiedad horizontal pueden convivir dos o más prestadores de servicios de telecomunicaciones [56].

El concepto de redes elásticas, permitirán a Colombia una mayor simplificación de la red interna de telecomunicaciones (ver Fig. 8) y aportará benéficamente de la siguiente manera: 1) Los Supercanales con el uso de múltiples subportadoras y los formatos de modulación avanzados consolidarán las demandas de una gran cantidad de usuarios en un solo Supercanal gracias a su propiedad de alta densidad y eficiencia espectral en un solo hilo de fibra óptica. 2) Las redes FiWi brindarán la posibilidad de centralizar gran parte de la infraestructura distribuida de un proveedor de servicios de telecomunicaciones en sus propias oficinas centrales y simplificar la topología integrando las redes metropolitana y de acceso. Por otro lado junto con la tecnología inalámbrica, además de la simplificación, se ahorrarán costos en el despliegue y mantenimiento de redes cableadas hasta el usuario final. 3) El concepto de redes elásticas aportará en el uso y despliegue eficiente de todos los recursos desde los propios de la red de transporte hasta los de la red integrada metro-acceso adaptándose a la demanda dinámica de información y mayor robustez ante fallas.

Los aportes mencionados facilitarán a Colombia el control sobre la infraestructura subyacente en las instalaciones de régimen de propiedad horizontal ya que cada proveedor, de llevar a cabo el despliegue de dichas tecnologías, reducirá su infraestructura y por ende, la complejidad de la red interna y facilitará el ingreso de otros prestadores de servicios de telecomunicaciones dando lugar a la libertad del usuario final de elegir a su proveedor e incentivar la libre competencia como lo estipula la comi- sión de regulación de comunicaciones.

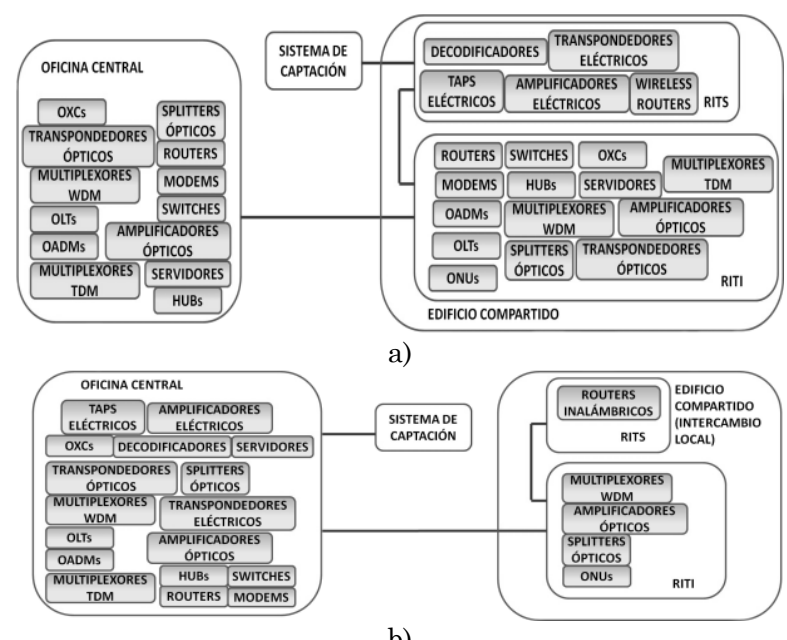

b)

Fig. 8. Simplificación de la red: a) Distribución de la infraestructura de la red actual, b) distribución de la infraestructura de una red basada en Fi-Wi. Fuente: Autores

\section{CONCLUSIONES}

Las telecomunicaciones de la siguiente generación permitirán una convergencia entre el dominio óptico e inalámbrico para brindar simplicidad, ancho de banda robusto y ubicuidad al mismo tiempo gracias a las virtudes que ofrecen las tecnologías en investigación como LR/PON, RoF y los Supercanales, lo cual será de mucha relevancia para las tareas de regulación de la red interna de telecomunicaciones. En la red de transporte, los Supercanales contribuirán con la transmisión masiva de información en anchos de banda muy reducidos y separaciones pequeñas en el dominio de la frecuencia lo cual permitirá la reutilización de las fibras ópticas ya desplegadas en el horizonte de tiempos estipulado por los entes reguladores de las telecomunicaciones en Colombia.

Todo lo anterior nos permite poner en perspectiva que en cada nivel de la red (red metro-acceso y transporte) habrá capacidad de reconfiguración de las redes de telecomunicaciones logrando un mejor aprovechamiento de la infraestructura subyacente en los edificios. 
Por tal motivo, Colombia con el despliegue masivo de redes de fibra óptica en los últimos años y con el programa nacional de fibra óptica donde se conectan más de 700 municipios, resulta un escenario viable a nivel técnico para probar y poner en marcha las redes elásticas. Para acelerar el desarrollo de las telecomunicaciones hacia un paradigma de redes elásticas, Colombia debe participar de manera activa en la investigación y desarrollo de nuevas tecnologías orientada a la habilitación de redes de próxima generación.

\section{AGRADECIMIENTOS}

Agradecemos a la Universidad de Antioquia y en especial al CODI (Comité para el Desarrollo de la Investigación) por su apoyo en nuestra investigación mediante el proyecto MDC11-1-04.

\section{REFERENCIAS}

[1] MINTIC, "Ley 1341 de 2009 "Por la cual se definen principios y conceptos sobre la sociedad de la información y la organización de las Tecnologías de la Información y las Comunicaciones -TIC-, se crea la Agencia Nacional del Espectro y se dictan otras disposiciones," Bogota-Colombia, 2009.

[2] M. del R. Guerra and J. D. Oviedo, "De las Telecomunicaciones a las TIC: Ley de TIC de Colombia (L1341/09)," 2011.

[3] I. Tomkos, E. Palkopoulou, and M. Angelou, "A survey of recent developments on flexible/elastic optical networking," in 2012 14th International Conference on Transparent Optical Networks (ICTON), 2012, pp. 1-6.

[4] O. Gerstel, M. Jinno, A. Lord, and S. J. Yoo, "Elastic optical networking: a new dawn for the optical layer?," IEEE Commun. Mag., vol. 50, no. 2, pp. s12-s20, Feb. 2012.

[5] Infinera, "Super-Channels: DWDM Transmission at 100Gb/s and Beyond," 2012.

[6] S. J. Savory, "Electronic signal processing in optical communications," in Proceedings SPIE, 2008, vol. 7136, p. 71362C-71362C-9.

[7] G. Colavolpe, T. Foggi, E. Forestieri, and G. Prati, "Robust Multilevel Coherent Optical Systems With Linear Processing at the
Receiver," J. Light. Technol., vol. 27, no. 13, pp. 2357-2369, Jul. 2009.

[8] D. van den Borne, M. Alfiad, S. L. Jansen, and T. Wuth, "40G/100G long-haul optical transmission system design using digital coherent receivers," in 2009 14th OptoElectronics and Communications Conference, 2009, pp. 1-2.

[9] G. Bosco, V. Curri, A. Carena, P. Poggiolini, and F. Forghieri, "On the Performance of NyquistWDM Terabit Superchannels Based on PMBPSK, PM-QPSK, PM-8QAM or PM-16QAM Subcarriers," J. Light. Technol., vol. 29, no. 1, pp. 53-61, Jan. 2011.

[10] R. Cigliutti, A. Nespola, D. Zeolla, G. Bosco, A. Carena, V. Curri, F. Forghieri, Y. Yamamoto, T. Sasaki, and P. Poggiolini, "Ultra-Long-Haul Transmission of $16 \times 112 \mathrm{~Gb} / \mathrm{s}$ SpectrallyEngineered DAC-Generated Nyquist-WDM PM16QAM Channels with 1.05x(Symbol-Rate) Frequency Spacing," in Optical Fiber Communication Conference (OFC), 2012, pp. 1-3.

[11] S.-J. Park, Y.-B. Choi, J.-M. Oh, S.-G. Koo, and D. Lee, "An Evolution Scenario of a Broadband Access Network Using R-SOA-Based WDM-PON Technologies," J. Light. Technol., vol. 25, no. 11, pp. 3479-3487, Nov. 2007.

[12]J. Kani, "Enabling Technologies for Future Scalable and Flexible WDM-PON and WDM/TDM-PON Systems," IEEE J. Sel. Top. Quantum Electron., vol. 16, no. 5, pp. 1290-1297, Sep. 2010.

[13] H. Kim, J. Hwang, and M. Yoo, "A Cost-Efficient WDM-PON Architecture Supporting Dynamic Wavelength and Time Slot Allocation," in The 9th International Conference on Advanced Communication Technology, 2007, pp. 15641568.

[14] G.-K. Chang, A. Chowdhury, Z. Jia, H.-C. Chien, M.-F. Huang, J. Yu, and G. Ellinas, "Key Technologies of WDM-PON for Future Converged Optical Broadband Access Networks [Invited]," J. Opt. Commun. Netw., vol. 1, no. 4, p. C35, Aug. 2009.

[15]M. E. Abdalla, S. M. Idrus, and A. B. Mohammad, "Hybrid TDM-WDM 10G-PON for high scalability next generation PON," in 2013 IEEE 8th Conference on Industrial Electronics and Applications (ICIEA), 2013, pp. 1448-1450.

[16] S. Bhandari and E. K. Park, "Hybrid Optical Wireless Networks," in International Conference on Networking, International Conference on Systems and International Conference on Mobile Communications and Learning Technologies (ICNICONSMCL'06), 2006, pp. 113-113.

[17]Z. M. Fadlullah, H. Nishiyama, N. Kato, H. Ujikawa, K.-I. Suzuki, and N. Yoshimoto, "Smart FiWi Networks: Challenges and Solutions for QoS and Green Communications," 
IEEE Intell. Syst., vol. 28, no. 2, pp. 86-91, Mar. 2013.

[18] G. Shen, R. Tucker, and C.--J. Chae, "Fixed Mobile Convergence Architectures for Broadband Access: Integration of EPON and WiMAX," IEEE Commun. Mag., vol. 45, no. 8, pp. 44-50, Aug. 2007.

[19] M. Maier, N. Ghazisaidi, and M. Reisslein, "The Audacity of Fiber-Wireless (FiWi) Networks," in in AccessNets, vol. 6, C. Wang, Ed. Berlin, Heidelberg: Springer Berlin Heidelberg, 2009, pp. 16-35.

[20] C. C. Davis, I. I. Smolyaninov, and S. D. Milner, "Flexible optical wireless links and networks," IEEE Commun. Mag., vol. 41, no. 3, pp. 51-57, Mar. 2003.

[21]G. Kramer, M. De Andrade, R. Roy, and P. Chowdhury, "Evolution of Optical Access Networks: Architectures and Capacity Upgrades," Proc. IEEE, vol. 100, no. 5, pp. 11881196, May 2012.

[22] H. Song, B.-W. Kim, and B. Mukherjee, "Longreach optical access networks: A survey of research challenges, demonstrations, and bandwidth assignment mechanisms," IEEE Commun. Surv. Tutorials, vol. 12, no. 1, pp. 112 123, 2010.

[23] G. Das, B. Lannoo, H.-D. Jung, T. Koonen, D. Colle, M. Pickavet, and P. Demeester, "A new architecture and MAC protocol for fully flexible hybrid WDM/TDM PON," in 2009 35th European Conference on Optical Communication, 2009, pp. 1-2.

[24] F. An, K. S. Kim, Y. Hsueh, M. Rogge, W. Shaw, and L. Kazovsky, "Evolution, challenges and enabling technologies for future WDM-based optical access networks," in Proc. of JCIS 2003, 2003, pp. 1449-1453.

[25] K. Iwatsuki, "Application and technical issues of WDM-PON," in Proceedings SPIE, 2010, vol. 7620, pp. 76200C-1-76200C-10.

[26] F. Clarke, S. Sarkar, and B. Mukherjee, "Simultaneous and interleaved polling: an upstream protocol for WDM-PON," in 2006 Optical Fiber Communication Conference and the National Fiber Optic Engineers Conference, 2006, pp. 1-3.

[27] D. Harle and I. Andonovic, "Dynamic bandwidth allocation algorithm for differentiated services over WDM EPONs," in The Ninth International Conference on Communications Systems, 2004, 2004, pp. 116-120.

[28] L. Zhou, X. Cheng, Y.-K. Yeo, and L. H. Ngoh, "Hybrid WDM-TDM PON architectures and DWBA algorithms," in 2010 5th International ICST Conference on Communications and Networking in China, 2010, pp. 1-6.

[29] D. P. Shea and J. E. Mitchell, "A 10-Gb/s 1024Way-Split 100-km Long-Reach Optical-Access
Network," J. Light. Technol., vol. 25, no. 3, pp. 685-693, Mar. 2007.

[30] M. O. van Deventer, J. D. Angelopoulos, H. Binsma, A. J. Boot, P. Crahay, E. Jaunart, P. J. M. Peters, A. J. Phillips, X.-Z. Qiu, J. M. Senior, M. Valvo, J. Vandewege, P. J. Vetter, and I. Van de Voorde, "Architectures for 100-km 2048 split bidirectional SuperPONs from ACTS-PLANET," in Proceedings SPIE, 1996, pp. 242-251.

[31] M. Oskar van Deventer, Y. M. van Dam, P. J. M. Peters, F. Vermaerke, and A. J. Phillips, "Evolution phases to an ultra broadband access network: results from ACTS-PLANET," IEEE Commun. Mag., vol. 35, no. 12, pp. 72-77, 1997.

[32] R. P. Davey, P. Healey, I. Hope, P. Watkinson, D. B. Payne, O. Marmur, J. Ruhmann, and Y. Zuiderveld, "DWDM reach extension of a GPON to $135 \mathrm{~km}$," in OFC/NFOEC Technical Digest. Optical Fiber Communication Conference, 2005, 2005, vol. 24, no. 1, p. 3 pp. Vol. 5.

[33] L. Kazovsky, S.-W. Wong, T. Ayhan, K. M. Albeyoglu, M. R. N. Ribeiro, and A. Shastri, "Hybrid Optical-Wireless Access Networks," Proc. IEEE, vol. 100, no. 5, pp. 1197-1225, May 2012.

[34] P. Chowdhury, M. Tornatore, S. Sarkar, and B. Mukherjee, "Building a Green Wireless-Optical Broadband Access Network (WOBAN)," J. Light. Technol., vol. 28, no. 16, pp. 2219-2229, Aug. 2010.

[35] N. Ghazisaidi, M. Maier, and C. Assi, "Fiberwireless (FiWi) access networks: A survey," IEEE Commun. Mag., vol. 47, no. 2, pp. 160-167, Feb. 2009.

[36]Z. Jia, J. Yu, and G. K. Chang, "A full-duplex radio-over-fiber system based on optical carrier suppression and reuse," IEEE Photonics Technol. Lett., vol. 18, no. 16, pp. 1726-1728, Aug. 2006.

[37]J. J. Granada, C. Serpa, G. Varón, and N. Guerrero, "Hacia la proxima generación de sistemas de radio sobre fibra de banda ancha: Retos tecnológicos en la banda de las ondas milimétricas," Rev. Científica Ing. y Desarro., vol. 29, no. 2, pp. 242-265, 2011.

[38] F.-M. Kuo, J.-W. Shi, H.-C. Chiang, H.-P. Chuang, H.-K. Chiou, C.-L. Pan, N.-W. Chen, H.J. Tsai, and C.-B. Huang, "Spectral Power Enhancement in a $100 \mathrm{GHz}$ Photonic MillimeterWave Generator Enabled by Spectral Line-byLine Pulse Shaping," IEEE Photonics J., vol. 2, no. 5, pp. 719-727, Oct. 2010.

[39]R. Sambaraju, J. Herrera, J. Marti, U. Westergren, D. Zibar, A. Caballero, J. B. Jensen, I. T. Monroy, and A. Walber, "Up to $40 \mathrm{~Gb} / \mathrm{s}$ wireless signal generation and demodulation in $75-110 \mathrm{GHz}$ band using photonic techniques," in 2010 IEEE International Topical Meeting on Microwave Photonics, 2010, pp. 1-4. 
[40] N. Cvijetic, M. Cvijetic, M.-F. Huang, E. Ip, Y.K. Huang, and T. Wang, "Terabit Optical Access Networks Based on WDM-OFDMA-PON," $J$. Light. Technol., vol. 30, no. 4, pp. 493-503, Feb. 2012.

[41] P. P. Biswas, A. Singh, and D. Chadha, "Energy efficient design for Green optical core network," in 2013 National Conference on Communications (NCC), 2013, pp. 1-5.

[42] G. Shen and Q. Yang, "From coarse grid to minigrid to gridless: How much can gridless help contentionless?," in 2011 Optical Fiber Communication Conference and Exposition and the National Fiber Optic Engineers Conference, 2011, pp. 1-3.

[43] S. Zhang and B. Mukherjee, "Energy-efficient dynamic provisioning for spectrum elastic optical networks," in 2012 IEEE International Conference on Communications (ICC), 2012, pp. 3031-3035.

[44] M. Angelou, D. Klonidis, K. Christodoulopoulos, A. Klekamp, F. Buchali, E. Varvarigos, and I. Tomkos, "Spectrum, Cost, and Energy Efficiency in Fixed-Grid and Flex-Grid Networks," in Optical Fiber Communication Conference and Exposition (OFC/NFOEC), 2012, pp. 1-3.

[45] L. Shi, S. Lee, H. Song, and B. Mukherjee, "Energy-Efficient Long-Reach Passive Optical Network: A Network Planning Approach Based on User Behaviors," IEEE Syst. J., vol. 4, no. 4, pp. 449-457, Dec. 2010.

[46]A. Ragheb and H. Fathallah, "Candidate modulation schemes for next generation-passive optical networks (NG-PONs)," in High Capacity Optical Networks and Emerging/Enabling Technologies, 2012, pp. 226-231.

[47] E. Lach and W. Idler, "Modulation formats for 100G and beyond," Opt. Fiber Technol., vol. 17, no. 5, pp. 377-386, Oct. 2011.

[48] G. Bosco, A. Carena, V. Curri, P. Poggiolini, and
F. Forghieri, "Performance Limits of NyquistWDM and CO-OFDM in High-Speed PM-QPSK Systems," IEEE Photonics Technol. Lett., vol. 22, no. 15, pp. 1129-1131, Aug. 2010.

[49] M. Klinkowski, M. Ruiz, L. Velasco, D. Careglio, V. Lopez, and J. Comellas, "Elastic Spectrum Allocation for Time-Varying Traffic in FlexGrid Optical Networks," IEEE J. Sel. Areas Commun., vol. 31, no. 1, pp. 26-38, Jan. 2013.

[50] L. G. Kazovsky, G. Kalogerakis, and W.-T. Shaw, "Homodyne Phase-Shift-Keying Systems: Past Challenges and Future Opportunities," J. Light. Technol., vol. 24, no. 12, pp. 4876-4884, Dec. 2006.

[51] B. Cao, D. P. Shea, and J. E. Mitchell, "Wavelength converting optical access network for 10Gbit/s PON," in Optical Network Design and Modeling (ONDM), 2011 15th International Conference on, 2011, pp. 1-6.

[52] M. Endo, "Optical Frequency Comb Generator Using a Single-Sideband Suppressed-Carrier Modulation in an Amplified Circulating Fiber Loop," Laser Phys., vol. 12, no. 4, pp. 679-683, 2002.

[53]A. F. Betancur Perez, I. K. V. Agudelo, A. M. C. Soto, and N. G. Gonzalez, "Broadband optical wavelenght conversion for next generation optical superchannels," in 2013 IEEE Colombian Conference on Communications and Computing (COLCOM), 2013, pp. 1-5.

[54]A. Nguyen, C. Porzi, S. Pinna, G. Contestabile, and A. Bogoni, "40 Gb/s All-Optical Selective Wavelength Shifter," in Conference on Lasers and Electro-Optics (CLEO), 2012, pp. 1-2.

[55] ICONTEC, "NTC 5797 - Telecomunicaciones. Infraestructura común de telecomunicaciones," 2010.

[56] CRC, "Redes Internas de Telecomunicaciones para el Acceso a Servicios de Telecomunicaciones," 2011. 